\title{
New therapeutic opportunities for Alzheimer disease
}

\section{Jae-Won Jang}

Department of Neurology, Kangwon National University Hospital, Kangwon National University School of Medicine, Chuncheon, Korea

Received: October 25, 2021

Revised: December 29, 2021

Accepted: December 30, 2021

Corresponding author:

Jae-Won Jang, MD, PhD

Department of Neurology,

Kangwon National University

Hospital, 156 Baengnyeong-ro,

Chuncheon 24289, Korea

Tel: +82-33-258-9174

Fax: +82-33-258-2103

E-mail: light26@kangwon.ac.kr
The number of Alzheimer disease (AD) is increasing worldwide and it is still the most common cause of dementia. However, there are limited treatment option with just symptomatic medications. Therefore new disease modifying drugs were in great need for many years for AD treatment. Recently, aducanumab which is beta amyloid targeted immunotherapy, has been approved by the US Food and Drug Administration for the treatment of AD. Although there are many questions to be answered, this would be the landmark for $A D$ drug that attempt to treat a neurodegenerative disease rather than just symptoms. In this review, I would like to discuss the current development status of dementia treatment, its limitations, and the future opportunity.

Keywords: Alzheimer disease; Therapeutics; Amyloid

\section{Introduction}

Alzheimer disease $(\mathrm{AD})$ is the most common cause of dementia worldwide and the total number of patients is increasing [1]. This can be defined as a slowly progressive neurodegenerative disease characterized by neuritic plaques and neurofibrillary tangles (NFTs) as a result of accumulation of amyloid-beta peptide in the brain that result in multiple cognitive dysfunctions as well as change in behavioural and emotional control [2]. AD is important public health problem because of high prevalence and subsequent socioeconomic costs. Until 2021, only 4 drugs have been approved for treating $\mathrm{AD}$ as three acetylcholinesterase inhibitors (AChEI) (e.g., donepezil, rivastigmine, and galantamine) and one N-methyl-D-aspartate receptors (NMDAR) [3]. Although significant scientific advances were made in $\mathrm{AD}$ research field, current $\mathrm{AD}$ drugs are all symptomatic that they just lessen the symptoms of the disease instead of prevent the progression of disease.

Based on the amyloid cascade hypothesis, amyloid-targeted treatment to reduce $\beta$-amyloid (A $\beta$ ) was expected to inhibit dis- ease progression or delay functional decline, but most clinical trials failed to prove its effectiveness [4]. However, aducanumab, which was recently conditionally approved by the US Food and Drug Administration (FDA) as an $\mathrm{AD}$ treatment, is a dementia drug approved 18 years after memantine in 2003, and unlike existing treatments, $A \beta$ target. Because of the possibility of changing the course of disease with drugs, it is receiving great attention and expectations from not only the medical community but also the general public. In this article, with the approval of aducanumab, we would like to investigate new therapeutic opportunities for $\mathrm{AD}$.

\section{Current pharmacologic therapeutic option}

Current FDA approved anti-AD drugs are based on the hypothesis of two neurotransmitters: acetylcholine and glutamate. The main action of the cholinesterase inhibitors is inhibition of cholinesterase, the enzyme which breaks down acetylcholine in brain synapses, elongating the effect of the decreased level of

Copyright (C) 2022 Korean Society of Geriatric Neurology

This is an Open Access article distributed under the terms of the Creative Commons Attribution Non-Commercial License (http://creativecommons.org/licenses/by-nc/4.0/) which permits unrestricted non-commercial use, distribution, and reproduction in any medium, provided the original work is properly cited. 
brain acetylcholine [5]. Three AChEI are currently available. Previous meta-analyses have revealed that these drugs delay cognitive dysfunction, slow the decline in global clinical dementia rating (CDR), and can delay the decline of activities of daily living about 6 to 12 months on average [6,7]. Side effects include gastrointestinal symptoms, vertigo, dizziness, insomnia, fatigue, bradycardia, and syncope [8]. In the course of $\mathrm{AD}$, overstimulation of glutamate in the brain lead to an excitotoxicity by calcium influx to neurons through NMDAR channels [9]. Excitotoxicity result in a gradual synaptic dysfunction and neurodegeneration in $\mathrm{AD}$. The NMDAR plays an essential role in synaptic plasticity and in glutamate synaptic transmission and related to learning and memory [10]. Memantine is a NMDAR antagonist and modulates it to reduce glutamate-induced excitotoxicity [11]. Memantine is FDA-indicated for moderate to severe $\mathrm{AD}$ [12] because it has improved stage of dementia assessment scores, global function assessment scores, activities of daily living scores [13] as well as neuropsychiatric symptoms such as delusions, disinhibition, and agitation [14].

\section{The current trends in AD drug research}

Development of AD-specific disease modifying therapy is continuing based on pathophysiology. According to 'Alzheimer's disease drug development pipeline: 2021' report by the Alzheimer's Association's, as of January 2021, 152 clinical trials for a total of 126 candidate substances are in progress, and majority of the total drugs (82.5\%) are disease-modifying drugs [15]. In terms of drug mechanism, it is expanding its scope to include tau, inflammation, neuroprotection, vascular factors, metabolism, and nerve regeneration as well as beta-amyloid [16].

\section{Emerging anti-amyloid therapies}

The monoclonal antibody as passive immunotherapeutic agents were developed after failure of vaccine for $\mathrm{AD}$ [17] to reduce effect of $A \beta 42$ in central as well as peripheral region. Although a few clinical trial of anti-amyloid immunotherapy have failed, some is undergoing. Aducanumab is a human anti-amyloid monoclonal antibody of which target is aggregated forms of soluble or insoluble $A \beta$. In a dose and time dependent manner, aducanumab enters the central nervous system as infusion that significantly decreases plaque in mild or prodromal $\mathrm{AD}$ that was confirmed by amyloid positron emission tomography (PET) [18]. Dose-related amyloid-related imaging abnormalities-ede$\mathrm{ma}$ (ARIA-E) are the main safety related finding and more common in Apo- $\varepsilon 4$ carriers. The infusion of highest dose of $10 \mathrm{mg} /$ $\mathrm{kg}$ was reported the significant decline the progression of cognitive impairment measured by the CDR-sum of boxes [18]. As a result, aducanumab was approved by the FDA for the treatment of $\mathrm{AD}$ with the condition that a phase IV trial carefully assess its safety and efficacy in June of 2021. This accelerated approval process for the use toward $\mathrm{AD}$-related mild cognitive impairment (MCI) or mild dementia raised some controversy related to limited evidence of treatment benefit. Recently, appropriate use of aducanumab by expert panel was released (Table 1) [19]. Therefore, patients should meet the clinical criteria for mild $\mathrm{AD}$ dementia or MCI due to $\mathrm{AD}$ supported by validated cognitive scores. Cholinesterase inhibitors or memantine can be used for these patients.

Amyloid status should be confirmed with an amyloid PET or cerebrospinal fluid (CSF) biomarkers. ApoE-є4 genotyping should be discussed with the patient/care giver for the risk of ARIA. A brain magnetic resonance imaging (MRI) should be obtained within 1 year before initiating treatment. Medical, cardiovascular, and psychiatric status should be stable before initiating treatment. Patients who are pregnant, on anticoagulant medications, or have evidence of significant cerebrovascular disease on brain MRI should be excluded from treatment. Patients and care partners should understand the requirements of therapy. Clinicians may need to collaborate with or refer to specialists with expertise in these assessments. This guideline also included dosing and MRI monitoring schedule as monthly infusion for a year [19].

Lecanemab or BAN2401 is IgG1 monoclonal antibody that binds to large soluble $A \beta$ protofibrils selectively. It has showed to increase CSF levels of $A \beta$, reduce total tau levels and slow cognitive decline, although further validation these findings is necessary [20]. The clinical trials for early $\mathrm{AD}$ or preclinical $\mathrm{AD}$ looking at efficacy and safety are in progress currently. Donanem$\mathrm{ab}$ is an immunoglobulin whose molecular target is cerebral amyloid plaque. For early $\mathrm{AD}$ subjects, some improvement in composite cognitive scores and activities of daily living score was achieved although asymptomatic ARIA-E were observed in phase 2 trial [21]. A safety and tolerability study showed $40 \%$ to $50 \%$ amyloid reduction and approximately $90 \%$ of participants developed anti-drug antibodies at 3 months after a single intravenous dose [22]. Gantenerumab is an another monoclonal antibody that has a higher affinity for $A \beta$ oligomers [23]. At higher doses, there was a reduction of amyloid plaque at two years by PET study [24]. Trials evaluating safety, tolerability, efficacy among early $\mathrm{AD}$ subjects or genetic $\mathrm{AD}$ are currently ongoing. 
Table 1. Appropriate use criteria for aducanumab in clinical practice

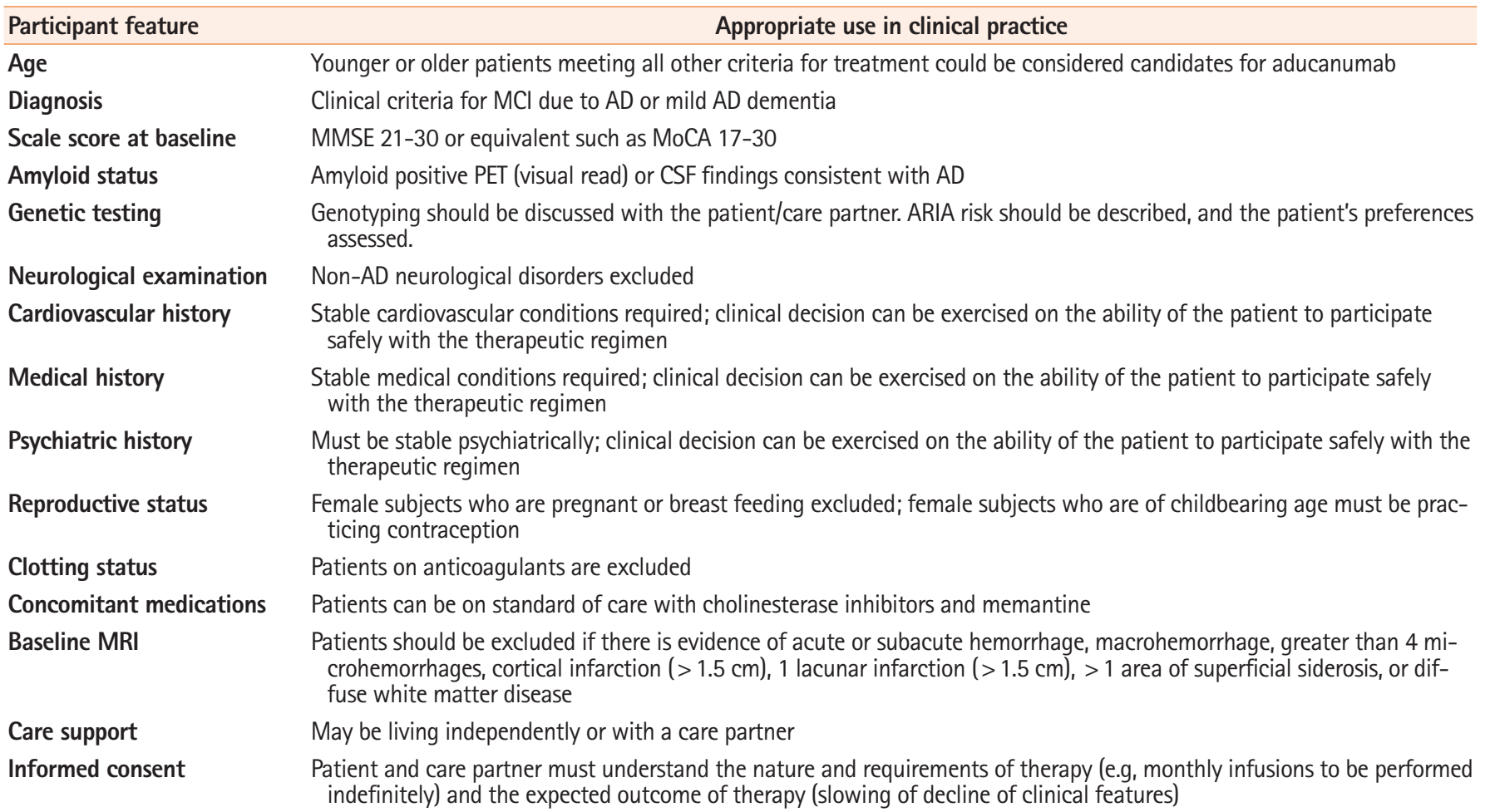

Adapted from Cummings J, et al. J Prev Alzheimers Dis 2021;8:398-410, according to the Creative Commons license [19].

$\mathrm{MCl}$, mild cognitive impairment; $\mathrm{AD}$, Alzheimer disease; MMSE, mini mental state examination; MoCA, montreal cognitive assessment; PET, positron emission tomography; CSF, cerebrospinal fluid; ARIA, amyloid-related imaging abnormalities; MRI, magnetic resonance imaging.

\section{Controversies in anti-amyloid therapies}

Following accelerated approval of aducanumab, FDA gave "breakthrough therapy designation" on lecanemab and donanem$\mathrm{ab}$ at June of 2021. This process is a way to accelerate the review and development of drugs underway for life-threatening or serious disease such as AD. This designation is not an indication that the drugs will be finally be affirmed to be safe and effective nor that they will be approved by FDA. Numerous randomized controlled trial studies based on the amyloid cascade reaction hypothesis so far have failed and it is argued that the discrepancy of aducanumab trials between the results of the EMERGE and ENGAGE clinical trials, which were designed with the same subject selection and research method, is derived from statistical error [25]. The difference between the results of the two trials might be derived from the difference in the ApoE- 44 allele, the method of dose increasing, and the fact that there were relatively many 'rapid progressors' among the high-dose patients who participated in the ENGAGE study although the definite standard for 'rapid progression' is not established [26]. In clinical trials, post hoc analysis is suitable for hypothesis setting, but not suitable for statistical validation. Therefore, there are issues that post hoc analysis is not recommended for FDA approval.

Regarding safety, ARIA-E and Amyloid-related imaging abnormalities-haemosiderin (ARIA-H) appear due to damage to the blood-brain barrier in the process of immune-mediated clearance of amyloid accumulated in the brain with anti-amyloid therapy [27]. Aducanumab and gantenerumab occurred in approximately $30 \%$, with ARIA-E occurring more frequently in the high-dose aducanumab group and lecanemab, which has a relatively low affinity for amyloid plaque, occurred in about $10 \%$ [27]. ARIA usually appears at the beginning of treatment and is mostly asymptomatic. However, $6 \%$ of high-dose treatment patients discontinued treatment due to side effects. Additionally, ARIA showed serious symptoms such as confusion, disorientation, gait disturbance, ataxia, visual impairment, headache, nausea, falls, and blurred vision in about $0.9 \%$. Because of these issues, it is insisted that insurance should not cover aducanumab as a treatment for $\mathrm{AD}$ until additional phase III trials prove clinical benefit of high-dose aducanumab in people with mild $\mathrm{AD}$ dementia or MCI due to $\mathrm{AD}$ [28]. To sum up, the debate on the correlation of reduction of beta amyloid and clinical improvement in $\mathrm{AD}$ will continue until a clinical trial for efficacy is over. In addition to it, application of aducanumab to those with $\mathrm{AD}$ 
and concomitant condition or those with atypical $\mathrm{AD}$ could be the candidate of future studies [29].

\section{Tau directed therapies}

The shortness of clear efficacy of amyloid-based therapeutics has led researchers to explore other targets [30]. Tau protein is another pathologic hallmark that defines $\mathrm{AD}$. It is a microtubule binding protein which forms NFTs and the accumulation of them have been known to correlate more significantly with severity of cognitive decline than amyloid load. There is evidence that $A \beta$ accumulation exacerbate tau pathology [31]. Tau protein has been found to mediate some of the toxic effects of $A \beta$ leading to dendritic simplification, synapse loss, and cell death in $\mathrm{AD}$ [32]. First approaches for tau directed therapies focused on inhibition of tau aggregation or kinases or stabilization of microtubules. Most of these studies have been stopped due to lack of efficacy or toxicity [33]. Tau immunotherapies are getting research focus currently. Variable targets are made related to tau targeting therapy because post-translational modifications and subsequent tau misfolding and loss of microtubule binding result in elevated levels of tau in the cytosol [32]. For example, the active tau vaccine AADvac1 represented a third of the participants were negative in tau-biomarker while clinical effect was not observed in the ADAMANT phase 2 study [34]. Therapeutic agents targeting tau protein, immunotherapy, tau aggregation inhibitors, and microtubule stabilizing substances are undergoing clinical trials and total of 11 drugs are included as of January of 2021 [15]. Compared to amyloid-related drugs, it is still in the early stages of research.

\section{Inflammation directed agents}

Lots of evidence has showed the involvement of microglia in $\mathrm{AD}$ pathogenesis and it is known to play both neuroprotective and neurotoxic effects $[35,36]$. Firstly, microglia with dual roles were designated as a pro-inflammatory M1 phenotype and immuno-suppressive M2 phenotype that can be changed under pathological and physiological conditions in the brain [37]. During the pleclinical $\mathrm{AD}$, microglia are activated by pro-inflammatory substances such as $A \beta$ and release inflammatory cytokines, including tumor necrosis factor- $\alpha$, interleukin (IL)- 6 , and $\mathrm{IL}-1 \beta$, and reactive oxygen that results in neuronal death and synaptic dysfunction [38]. There are increasing trend in inflammation directed agents that have multiple targets such as microglial activation, stabilization of mast cell, T-cell regulation and reduction of microbiome dystosis [15]. There are 2 phase 3 trials, 12 phase 2 trials and 5 phase 1 trials related inflammation/infection/immunity as of January, 2021.

\section{Metabolism and bioenergetics}

Metabolic perturbations have been known to be linked to $\mathrm{AD}$ both at the cellular level in the brain as well as in the whole body [1]. Type two diabetes increases the risk of $\mathrm{AD}[39,40]$ and insulin sensitivity is impaired in the majority of elderly people [41]. Previous studies related to epidemiology, mechanisms of disease and clinical manifestations revealed links between $\mathrm{AD}$ and diabetes $[2,42-45]$. Metabolic pathways leading to energy production are essential for normal neuronal function and the brain of $\mathrm{AD}$ was related to decreased glucose metabolism [42]. Although the underlying molecular mechanisms between diabetes and $\mathrm{AD}$ are not yet obvious [46], anti-diabetic drugs are reviewed for clinical benefit in $\mathrm{AD}$ [47-49]. Additionally, disruption of glucose metabolism and mitochondrial dysfunction are a common feature of both $\mathrm{AD}$ and diabetes [50]. Because of the essential role of mitochondria in bioenergetics, targeting the mitochondrial function has emerged as a therapeutic strategy for neurodegenerative diseases such as $\mathrm{AD}$ [51-53]. Emerging targets for this area are insulin sensitizer, glucagon-like peptide receptor agonist, mitochondrial enhancers and ketosis-inducing agents. There are 2 phase 3 trials, 4 phase 2 trials and 15 phase 1 trials related metabolism and bioenergetics as of January, 2021 [15].

\section{Conclusion}

The research experience of monoclonal antibody therapeutics against amyloid beta has led to increasing interest in therapeutic approaches based on disease modifying agents targeting amyloid beta hypothesis in the brain, as well as other targets, such as tau, inflammation and synaptic dysfunction. If there is a lesson learned at a high expense from the failure and success of existing drugs, biological targets must be identified through biomarkers such as PET or CSF in addition to clinical diagnosis when recruiting patients, and drugs required higher doses than initially expected. It can be suggested that patients with mild symptoms had better drug responsiveness, and that a more sensitive cognitive function measurement tool was needed to confirm the effect of the drug. In 2018, the Research Framework, jointly announced by the National Institute of Aging and Alzheimer's Association, defines AD only with biomarkers [54]. It is expected to play a very important role in confirming the effectiveness of treatment. Biomarkers mainly used include amyloid and tau in CSF, brain MRI, and amyloid 
PET, and the use of blood amyloid and tau and tau PET is expected to gradually increase. In fact, the drug development guidelines published in February 2018 by the US FDA emphasize biomarker-based clinical trials in early patients [55]. After decades of development and research of biomarkers in $\mathrm{AD}$, they are having a substantial impact on the development of disease-modifying drugs [54]. Biomarkers were essential to the approval of aducanumab, and are used in other studies for the assessment of treatment efficacy and target engagement and patient selection. Although molecular imaging of beta amyloid and tau were commonly used in clinical trials, the need for high accessibility and reliability for diverse populations was increased a lot this year. Therefore the studies on the use of blood-based biomarkers was validated against molecular imaging that p-tau 217 and p-tau181 represented high performance for differentiating $\mathrm{AD}$ with other neurodegenerative disease $[56,57]$. Ultimately, the success of future drug research depends on research innovation that sets the most important targets for the disease process, develops effective drugs for these targets, and conducts rigorous clinical trials. In line with this research trend, $\mathrm{AD}$ has been the driving force in the development of new drugs due to the social demand and responsibility due to the limitations of the treatment effect and choice. The recent FDA approval of aducanumab opens a new era in the treatment of $\mathrm{AD}$, bringing interest and hope to patients, families, and healthcare workers facing an aging population. Although there are many controversies to be resolved in the future, the approval of aducanumab will affect and change the approval of future treatments. Physicians must have an ethical responsibility to fully explain to patients and their families not only the effects of drugs, but also aspects of safety or uncertainties. That is to say, clinicians should be well aware of side effect of aducanumab such as ARIA-related symptoms. The debate on whether reduction of $\mathrm{A} \beta$ correlates clinical improvement in $\mathrm{AD}$ continues and the cost of this medication is high as much as more than 50 thousand dollars annually. On top of that, further studies are needed to assess if aducanumab is appropriate for those with other disease beyond $\mathrm{MCI}$ due to $\mathrm{AD}$ or $\mathrm{AD}$ dementia. $\mathrm{Be}$ cause many questions that should be solved remain, clinicians also need to be well informed about this new drug as upcoming promising therapeutic opportunity.

\section{Notes}

\section{Conflicts of Interest}

The author have no potential conflicts of interest to disclose.

\section{Funding}

None.

\section{ORCID}

Jae-Won Jang, https://orcid.org/0000-0003-3540-530X

\section{References}

1.2021 Alzheimer's disease facts and figures. Alzheimers Dement 2021;17:327-406.

2. De-Paula VJ, Radanovic M, Diniz BS, Forlenza OV. Alzheimer's disease. Subcell Biochem 2012;65:329-352.

3. Chiang K, Koo EH. Emerging therapeutics for Alzheimer's disease. Annu Rev Pharmacol Toxicol 2014;54:381-405.

4. Bomasang-Layno E, Bronsther R. Diagnosis and treatment of Alzheimer's disease: an update. Dela J Public Health 2021; 7:74-85

5. Ritchie CW, Zhinchin G. Low dose, high dose, or no dose: better prescribing of cholinesterase inhibitors for Alzheimer's disease. Int Psychogeriatr 2013;25:511-515.

6. Birks JS, Harvey RJ. Donepezil for dementia due to Alzheimer's disease. Cochrane Database Syst Rev 2018;6:CD001190.

7. Hansen RA, Gartlehner G, Webb AP, Morgan LC, Moore CG, Jonas DE. Efficacy and safety of donepezil, galantamine, and rivastigmine for the treatment of Alzheimer's disease: a systematic review and meta-analysis. Clin Interv Aging 2008;3:211225.

8. Joe E, Ringman JM. Cognitive symptoms of Alzheimer's disease: clinical management and prevention. BMJ 2019;367: 16217.

9. Sucher NJ, Awobuluyi M, Choi YB, Lipton SA. NMDA receptors: from genes to channels. Trends Pharmacol Sci 1996;17: 348-355.

10. Liu J, Chang L, Song Y, Li H, Wu Y. The role of NMDA receptors in Alzheimer's disease. Front Neurosci 2019;13:43.

11. Wang R, Reddy PH. Role of glutamate and NMDA receptors in Alzheimer's disease. J Alzheimers Dis 2017;57:1041-1048.

12. Reisberg B, Doody R, Stoffler A, Schmitt F, Ferris S, Mobius $\mathrm{HJ}$, et al. Memantine in moderate-to-severe Alzheimer's disease. N Engl J Med 2003;348:1333-1341.

13. McShane R, Westby MJ, Roberts E, Minakaran N, Schneider L, Farrimond LE, et al. Memantine for dementia. Cochrane Database Syst Rev 2019;3:CD003154.

14. Kishi T, Matsunaga S, Iwata N. The effects of memantine on behavioral disturbances in patients with Alzheimer's disease: a meta-analysis. Neuropsychiatr Dis Treat 2017;13:1909-1928.

15. Cummings J, Lee G, Zhong K, Fonseca J, Taghva K. Alzhei- 
mer's disease drug development pipeline: 2021. Alzheimers Dement (NY) 2021;7:e12179.

16. International Alzheimer's and Related Dementias Research Portfolio (IADRP). Common Alzheimer's and Related Dementias Research Ontology (CADRO) [Internet]. IADRP; 2020 [cited 2021 Oct 17]. Available from: https://iadrp.nia. nih.gov/about/cadro.

17. Lozupone M, Solfrizzi V, D’Urso F, Di Gioia I, Sardone R, Dibello V, et al. Anti-amyloid- $\beta$ protein agents for the treatment of Alzheimer's disease: an update on emerging drugs. Expert Opin Emerg Drugs 2020;25:319-335.

18. Sevigny J, Chiao P, Bussiere T, Weinreb PH, Williams L, Maier $\mathrm{M}$, et al. The antibody aducanumab reduces $\mathrm{A} \beta$ plaques in $\mathrm{Alz}$ heimer's disease. Nature 2016;537:50-56.

19. Cummings J, Aisen P, Apostolova LG, Atri A, Salloway S, Weiner M. Aducanumab: appropriate use recommendations. J Prev Alzheimers Dis 2021;8:398-410.

20. Loera-Valencia R, Cedazo-Minguez A, Kenigsberg PA, Page G, Duarte AI, Giusti P, et al. Current and emerging avenues for Alzheimer's disease drug targets. J Intern Med 2019;286:398437.

21. Mintun MA, Lo AC, Duggan Evans C, Wessels AM, Ardayfio PA, Andersen SW, et al. Donanemab in Early Alzheimer's Disease. N Engl J Med 2021;384:1691-1704.

22. Lowe SL, Willis BA, Hawdon A, Natanegara F, Chua L, Foster J, et al. Donanemab (LY3002813) dose-escalation study in Alzheimer's disease. Alzheimers Dement (NY) 2021;7:e12112.

23. Bohrmann B, Baumann K, Benz J, Gerber F, Huber W, Knoflach F, et al. Gantenerumab: a novel human anti-A $\beta$ antibody demonstrates sustained cerebral amyloid- $\beta$ binding and elicits cell-mediated removal of human amyloid- $\beta$. J Alzheimers Dis 2012;28:49-69.

24. Klein G, Delmar P, Voyle N, Rehal S, Hofmann C, Abi-Saab D, et al. Gantenerumab reduces amyloid- $\beta$ plaques in patients with prodromal to moderate Alzheimer's disease: a PET substudy interim analysis. Alzheimers Res Ther 2019;11:101.

25. Alexander GC, Emerson S, Kesselheim AS. Evaluation of aducanumab for Alzheimer disease: scientific evidence and regulatory review involving efficacy, safety, and futility. JAMA 2021; 325:1717-1718.

26. Lee AY. Amyloid-targeting drugs for the treatment of Alzheimer disease.J Korean Neurol Assoc 2021;39:134-140.

27. Tolar M, Hey J, Power A, Abushakra S. Neurotoxic soluble amyloid oligomers drive Alzheimer's pathogenesis and represent a clinically validated target for slowing disease progression. Int J Mol Sci 2021;22:6355.

28. Moghavem N, Henderson VW, Greicius MD. Medicare should not cover aducanumab as a treatment for Alzheimer's disease. Ann Neurol 2021;90:331-333.

29. Molano JR. The aducanumab controversy-how do clinicians proceed? [Internet]. NEJM J Watch; 2021 [cited 2021 Oct 11]. Available from: https://www.jwatch.org/NA54036/2021/ 09/29/aducanumab-controversy-how-do-clinicians-proceed.

30. Congdon EE, Sigurdsson EM. Tau-targeting therapies for Alzheimer disease. Nat Rev Neurol 2018;14:399-415.

31. Plotkin SS, Cashman NR. Passive immunotherapies targeting $A \beta$ and tau in Alzheimer's disease. Neurobiol Dis 2020;144: 105010.

32. Bakota L, Brandt R. Tau biology and tau-directed therapies for Alzheimer's disease. Drugs 2016;76:301-313.

33. Congdon EE, Wu JW, Myeku N, Figueroa YH, Herman M, Marinec PS, et al. Methylthioninium chloride (methylene blue) induces autophagy and attenuates tauopathy in vitro and in vivo. Autophagy 2012;8:609-622.

34. Novak P, Kovacech B, Katina S, Schmidt R, Scheltens P, Kontsekova E, et al. ADAMANT: a placebo-controlled randomized phase 2 study of AADvac1, an active immunotherapy against pathological tau in Alzheimer's disease. Nat Aging 2021;1: 521-534.

35. Qiao O, Ji H, Zhang Y, Zhang X, Zhang X, Liu N, et al. New insights in drug development for Alzheimer's disease based on microglia function. Biomed Pharmacother 2021;140:111703.

36. Hansen DV, Hanson JE, Sheng M. Microglia in Alzheimer's disease. J Cell Biol 2018;217:459-472.

37. Kierdorf K, Erny D, Goldmann T, Sander V, Schulz C, Perdiguero EG, et al. Microglia emerge from erythromyeloid precursors via Pu.1- and Irf8-dependent pathways. Nat Neurosci 2013;16:273-280.

38. Morris GP, Clark IA, Zinn R, Vissel B. Microglia: a new frontier for synaptic plasticity, learning and memory, and neurodegenerative disease research. Neurobiol Learn Mem 2013;105:4053.

39. Vieira M, Lima-Filho R, De Felice FG. Connecting Alzheimer's disease to diabetes: underlying mechanisms and potential therapeutic targets. Neuropharmacology 2018;136(Pt B):160171.

40. Cheng G, Huang C, Deng H, Wang H. Diabetes as a risk factor for dementia and mild cognitive impairment: a meta-analysis of longitudinal studies. Intern Med J 2012;42:484-491.

41. Hoyer S. Glucose metabolism and insulin receptor signal transduction in Alzheimer disease. Eur J Pharmacol 2004;490:115125.

42. Holscher C. Diabetes as a risk factor for Alzheimer's disease: insulin signalling impairment in the brain as an alternative 
model of Alzheimer's disease. Biochem Soc Trans 2011;39: 891-897.

43. Szablewski L. Glucose transporters in brain: in health and in Alzheimer's disease. J Alzheimers Dis 2017;55:1307-1320.

44. Kandimalla R, Thirumala V, Reddy PH. Is Alzheimer's disease a type 3 diabetes? A critical appraisal. Biochim Biophys Acta Mol Basis Dis 2017;1863:1078-1089.

45. Kubis-Kubiak AM, Rorbach-Dolata A, Piwowar A. Crucial players in Alzheimer's disease and diabetes mellitus: Friends or foes? Mech Ageing Dev 2019;181:7-21.

46. Salas IH, De Strooper B. Diabetes and Alzheimer's disease: a link not as simple as it seems. Neurochem Res 2019;44:12711278.

47. Meng L, Li XY, Shen L, Ji HF. Type 2 diabetes mellitus drugs for Alzheimer's disease: current evidence and therapeutic opportunities. Trends Mol Med 2020;26:597-614.

48. Boccardi V, Murasecco I, Mecocci P. Diabetes drugs in the fight against Alzheimer's disease. Ageing Res Rev 2019;54:100936.

49. Holscher C. Brain insulin resistance: role in neurodegenerative disease and potential for targeting. Expert Opin Investig Drugs 2020;29:333-348.

50. Rigotto G, Basso E. Mitochondrial dysfunctions: a thread sewing together Alzheimer's disease, diabetes, and obesity. Oxid Med Cell Longev 2019;2019:7210892.

51. Cunnane SC, Trushina E, Morland C, Prigione A, Casadesus G, Andrews ZB, et al. Brain energy rescue: an emerging therapeutic concept for neurodegenerative disorders of ageing. Nat Rev
Drug Discov 2020;19:609-633.

52. Fang EF, Hou Y, Palikaras K, Adriaanse BA, Kerr JS, Yang B, et al. Mitophagy inhibits amyloid- $\beta$ and tau pathology and reverses cognitive deficits in models of Alzheimer's disease. Nat Neurosci 2019;22:401-412.

53. Hill BG, Shiva S, Ballinger S, Zhang J, Darley-Usmar VM. Bioenergetics and translational metabolism: implications for genetics, physiology and precision medicine. Biol Chem 2019; 401:3-29.

54. Jack CR Jr, Bennett DA, Blennow K, Carrillo MC, Dunn B, Haeberlein SB, et al. NIA-AA Research Framework: toward a biological definition of Alzheimer's disease. Alzheimers Dement 2018; 14:535-562.

55. Sabbagh MN, Hendrix S, Harrison JE. FDA position statement "Early Alzheimer's disease: Developing drugs for treatment, Guidance for Industry”. Alzheimers Dement (N Y) 2019;5: 13-19.

56. Leuzy A, Janelidze S, Mattsson-Carlgren N, Palmqvist S, Jacobs D, Cicognola C, et al. Comparing the clinical utility and diagnostic performance of CSF P-Tau181, P-Tau217, and P-Tau231 assays. Neurology 2021;97:e1681-e1694.

57. Thijssen EH, La Joie R, Strom A, Fonseca C, Iaccarino L, Wolf A, et al. Plasma phosphorylated tau 217 and phosphorylated tau 181 as biomarkers in Alzheimer's disease and frontotemporal lobar degeneration: a retrospective diagnostic performance study. Lancet Neurol 2021;20:739-752. 